\title{
Cholestatic Hepatitis C in Immunocompromised Settings
}

\author{
Isabel A. Zacharias, MD, and Fredric D. Gordon, MD
}

\author{
Corresponding author \\ Fredric D. Gordon, MD \\ Department of Transplantation, Lahey Clinic Medical Center, \\ 41 Mall Road, 4 West, Burlington, MA 01805, USA. \\ E-mail: Fredric_D_Gordon@Lahey.org \\ Current Hepatitis Reports 2009, 8:148-152 \\ Current Medicine Group LLC ISSN 1540-3416 \\ Copyright $@ 2009$ by Current Medicine Group LLC
}

The hepatitis $\mathrm{C}$ virus (HCV) is a major public health concern and a leading cause of chronic liver disease and liver transplantation. If the virus is not eradicated before liver transplantation, HCV infection can recur in almost all patients. Recurrent $\mathrm{HCV}$ after liver transplantation has a variable clinical course. Rarely, a severe cholestatic form of recurrent HCV can occur, better known as fibrosing cholestatic hepatitis $(\mathrm{FCH})$. This type of cholestasis can lead to fibrosis and graft dysfunction in an accelerated fashion and is associated with a high morbidity and mortality. It was described in other organ transplant recipients and patients with HIV/ HCV coinfection, suggesting that an immunocompromised state is necessary to develop FCH.

\section{Introduction}

Cirrhosis caused by the hepatitis $\mathrm{C}$ virus (HCV) is the leading indication for liver transplantation among adults in the United States and Europe [1]. Recurrent HCV after liver transplantation is nearly universal and has serious consequences, including cirrhosis, graft failure, and death. The rate of progression of recurrent HCV after liver transplantation toward cirrhosis and liver failure is accelerated as compared with immunocompetent individuals [1-3]. In some cases, a progressive cholestatic form of recurrent HCV occurs, often leading to rapid graft dysfunction as early as 5 months after liver transplantation [4]. The frequency of this type of aggressive cholestasis in recurrent HCV is reported to be about $8 \%$ to $13 \%$ [5-7]. Fibrosing cholestatic hepatitis $(\mathrm{FCH})$ was first described in the early 1990 s in a liver transplant recipient with hepatitis $B$ virus (HBV), but is now rarely seen in this disease because of improved strategies for preventing HBV reinfection in liver allograft [8]. Since then, it has been described in patients with HCV recurrence after hepatic and nonhepatic organ transplants, and in patients coinfected with HIV and HCV [4,9-13], all of whom share the common bond of an immunocompromised state.

\section{Clinical Course of FCH After}

\section{Liver Transplantation}

FCH typically follows a subclinical course with the ominous consequence of graft failure and death. FCH is characterized by progressive jaundice with rapid deterioration of liver function that can result in ascites, portosystemic encephalopathy, variceal bleeding, coagulopathy, and death within 4 to 6 weeks of onset [14]. Laboratory testing reveals marked hyperbilirubinemia, prolonged international ratio, mild to moderate elevations of the liver transaminases, and markedly elevated HCV RNA levels in the bloodstream and liver tissue.

Schluger et al. [4] found that in eight patients with $\mathrm{FCH}$, the time from transplant to evidence of recurrent $\mathrm{HCV}$ infection was 5.4 months, the time from recurrent HCV infection to cholestasis was 5.3 months, and the time from cholestasis to retransplantation was 4.1 months. Evaluation of the explanted livers in all eight patients showed severe cholestasis and various degrees of fibrosis.

\section{Risk Factors for Development of \\ FCH After Liver Transplant}

Several factors may be associated with severe cholestatic HCV recurrence after liver transplantation.

\section{Viral factors}

High pretransplantation serum HCV RNA levels $\left(>10^{6}\right.$ $\mathrm{IU} / \mathrm{mL}$ ) predict the severity of recurrent $\mathrm{HCV}$ and rate of fibrosis progression associated with increased morbidity and mortality [15-17]. This high level of HCV RNA indicates markedly enhanced replication of $\mathrm{HCV}$, leading to direct cellular degeneration and death [13]. HCV genotype $1 \mathrm{~b}$ was reported to have a more frequent and severe form of recurrent HCV compared with other genotypes, but these results are conflicting $[4,10,18]$. A very high expression of HCV proteins in the liver graft was also thought to be linked to the development of FCH [19]. 


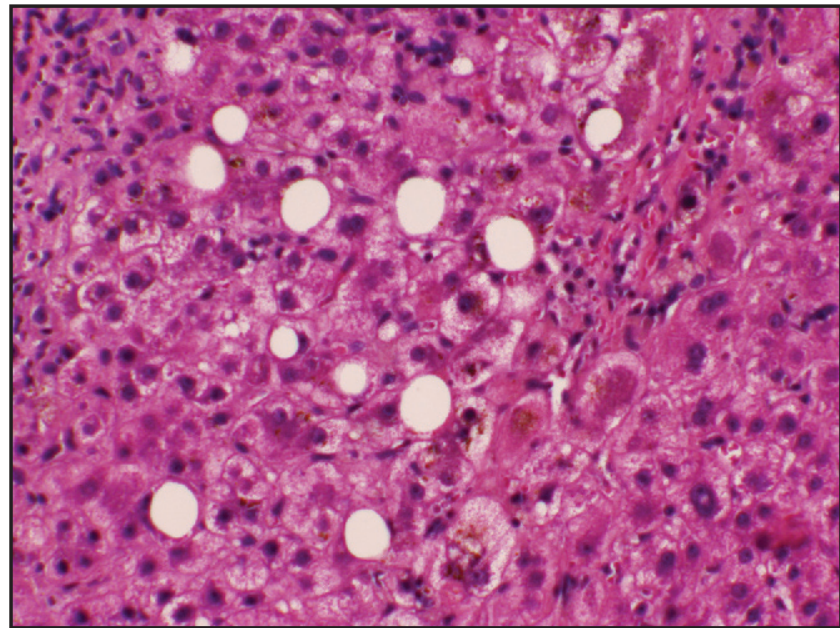

Figure 1. Photomicrograph of liver demonstrating hepatocanalicular cholestasis in a transplant recipient with fibrosing cholestatic hepatitis C. (Courtesy of Urmila Khettry, MD, Lahey Clinic Medical Center, Burlington, MA.)

\section{Immunosuppression}

FCH can occur in a variety of nonhepatic transplant recipients, suggesting the crucial role of immunosuppression in its pathogenesis. The theory of quasispecies patterns remains controversial. Some studies suggest immunosuppression enhances divergence of the HCV quasispecies and results in the emergence of new variants [19-22]. Other studies show that after transplantation, the quasispecies pattern remains stable over time in patients with recurrent cholestatic hepatitis HCV, which suggests that viral escape from immune pressure may play a pathogenic role [23].

\section{Pathogenesis of FCH}

The precise pathogenetic mechanisms behind $\mathrm{FCH}$ remain obscure. High levels of serum HCV RNA may lead to "direct-hit" cellular injury and rapid onset of graft dysfunction, which may explain the marked hyperbilirubinemia and only modest transaminitis often seen with $\mathrm{FCH}$. The direct, sudden, diffuse hepatocyte injury is reflected in the hepatocytic ballooning that occurs in much the same manner as alcohol-induced liver damage [24•].

\section{Histopathologic Findings in FCH}

Histologically, FCH is characterized by extensive dense portal fibrosis, immature fibrous bands extending into sinusoidal spaces, ductular proliferation and hypercellularity, marked canalicular and cellular cholestasis, hepatocytic ballooning, and mild to moderate inflammation (Fig. 1) [25]. In FCH related to HCV, the inflammatory infiltrate may consist predominantly of neutrophils [24•], compared with the mononuclear infiltration described in FCH related to HBV recurrence [25].

Dixon and Crawford [26••] developed a novel "sinusoidal system" to identify early patterns of histologic changes in four patients with FCH. In FCH, the earliest histologic abnormality to appear was mild, recurrent $\mathrm{HCV}$ and cholestasis. Cholestasis in recurrent HCV is atypical and should raise suspicion for the development of $\mathrm{FCH}$, even if the cholestasis is mild. Early suspicion and recognition of this aggressive entity is necessary. Cholestasis can be seen in acute cellular rejection (ACR) and chronic rejection with histologic patterns of endotheliitis and hepatic artery foam cell changes, respectively, which help to differentiate them from FCH. The cholestatic changes in FCH progress over several weeks. Fibrosis lags behind cholestasis by a few weeks. Early sinusoidal fibrotic changes were described as delicate pericellular bands that gradually extend until the classic fibrosis pattern becomes obvious. Fibrosis should not typically be seen in early cases of recurrent HCV alone or with rejection.

\section{HIV/HCV Coinfection}

The risk for developing $\mathrm{FCH}$ is increased in patients with HCV coinfected with HIV after liver transplantation. De Vera et al. [27•] examined the outcomes after liver transplantation in 27 HIV-positive patients who developed recurrent HCV. HIV infection was well controlled after transplantation. Recurrent HCV in 18 patients was associated with eight deaths, including three from $\mathrm{FCH}(37.5 \%)$.

FCH was also described in patients with HCV/HIV coinfection who have not undergone liver transplantation. This finding is an important clinical issue because about $30 \%$ of patients infected with HIV in the United States and Europe are also infected with HCV [28-30]. This rate of coinfection is not surprising given that HIV and HCV are acquired through similar routes, although at different rates. With the introduction of highly active antiretroviral therapy (HAART) in 1996, HIV mortality declined and HCVrelated liver disease is now the leading cause of morbidity and mortality in HIV-infected persons [31]. Progression of liver disease in patients with HIV/HCV coinfection is also accelerated. Unlike HCV recurrence after liver transplantation, in patients with HIV/HCV coinfection, HCV RNA levels are not markedly elevated, suggesting that direct cytopathic liver injury is not the sole cause of FCH and resultant liver dysfunction in this population. Rosenberg et al. [32] described two patients with HIV/HCV coinfection and found HCV RNA levels were about 1 million copies/mL, which are lower than HCV RNA levels typically described in patients with FCH after liver transplantation. Another theory proposed by this group for the onset of FCH in patients coinfected with HIV/HCV is the acute hepatitis flare, which may be seen after reconstitution of the immune system with the successful treatment of HIV disease using HAART. The role of antiviral treatment of HCV-induced FCH remains unclear in this population.

\section{Differential Diagnosis}

Several other posttransplant etiologies can cause cholestasis and graft dysfunction. Ischemic injury from delayed 
hepatic artery thrombosis, adverse drug reactions, ischemic reperfusion injury, and extrahepatic biliary obstruction can all cause hepatocytic ballooning, cholestasis, and Kupffer cell clustering [25]. Imaging studies of the hepatic vasculature and careful review of administered medications are essential, and the location of neutrophilic infiltration may help differentiate ascending cholangitis (neutrophils within lumen of the bile ducts) from FCH (neutrophils at the interface between portal tracts and lobules).

Differentiating ACR from FCH is often difficult, and can lead to erroneous diagnosis and therefore devastating outcomes with treatment. The presence of endotheliitis may help differentiate ACR from HCV recurrence [26••]. Neff et al. [33] found that identifying the typical FCH clinical presentation was better able to accurately predict cholestatic outcome than histologic parameters. Misdiagnosis of recurrent HCV as ACR on liver biopsy can lead to devastating consequences from the treatment, allowing for the continued severe course of liver dysfunction and patient death. Corticosteroid boluses, used perioperatively and for treatment of ACR, can negatively impact the severity of recurrent HCV [34]. Sheiner et al. [35] showed that muromonab (OKT3), used for steroid refractory rejection, increased the incidence and clinical severity of recurrent HCV $(71.4 \%$ compared with $37.3 \%$ of patients who did not require OKT3). Also, the time to HCV recurrence was accelerated from 246 days to 127 days.

\section{Treatment of FCH}

Early recognition of $\mathrm{FCH}$ is critical because of the rapid deterioration of liver function that ensues. Even with early, aggressive treatment, the outcome in patients with $\mathrm{FCH}$ is often poor. A decrease, or even discontinuation, of immunosuppression may result in a decrease in $\mathrm{HCV}$ RNA levels and therefore reduce ongoing liver damage, but at the risk of developing ACR.

Several studies evaluated the effect of interferon (IFN) monotherapy in patients with recurrent $\mathrm{HCV}$ after liver transplantation, and found no histologic improvement or HCV RNA clearance [36-38]. Only one patient of 11 in one study had a biochemical response [36]. No biochemical response occurred in any of the 18 patients in a second study [37]. Another study showed a biochemical response rate of $23 \%$ and a reduction in HCV RNA levels by $57 \%$ in 14 patients on IFN monotherapy treated for 6 months [38]. Ribavirin monotherapy was used for HCV recurrence after liver transplantation; it was not effective in HCV RNA clearance and caused only mild histologic improvement $[39,40]$.

Because of the improved results of combination IFN and ribavirin therapy in immunocompetent HCV patients, combination interferon and ribavirin were used to treat recurrent HCV after liver transplantation, although often in the setting of mild recurrence [41]. Gopal et al. [42] studied the safety and efficacy of antiviral treatment in 12 patients who developed moderate to severe HCV recurrence after transplantation. Most patients required dose reduction of both medications, and granulocyte colonystimulating factor was initiated in seven patients. Overall, serum biochemical markers decreased in the patients after treatment. Six of 12 patients became HCV RNA negative, although sustained virologic response was observed in only one patient. Five patients died, three of whom had progressive allograft failure from severe HCV recurrence.

Later, Gopal and Rosen [43] found that the duration of combination IFN and ribavirin could need to be indefinite in the subset of patients with severe cholestatic HCV recurrence. Seven patients with cholestatic HCV recurrence were treated with combination IFN and ribavirin therapy, initially with IFN administered three times a week, then switching to pegylated (PEG) IFN after 6 to 12 weeks, if tolerated. Four patients cleared the HCV RNA; however, discontinuation of therapy resulted in a rapid rebound in serum bilirubin and viral replication, and ultimately death. A case report of a patient who achieved resolution of FCH using combination PEG IFN and ribavirin despite discontinuation of therapy (because of profound depression) may suggest that the use of PEG IFN at the start of treatment decreases the risk for rebound FCH and death [44•]. The duration of therapy remains controversial, although long-term therapy may be necessary until further studies using PEG IFN with ribavirin become available.

Preemptive therapy before the development of HCV recurrence has been attempted with poor results [45]; therefore, a common approach is to treat with combination therapy after clinicopathologic recurrence was developed [46•]. Double-filtration plasmapheresis (DFPP) combined with IFN and ribavirin was used in a patient with HCV and a liver transplant, who later recovered from FCH [47•]. Several challenges to therapy remain, including optimal time to initiate therapy, length of therapy, and the significant side effects of therapy (ie, cytopenia), which are common in liver transplant patients in the first year of surgical recovery.

\section{Retransplantation for $\mathrm{FCH}$}

Increasing numbers of $\mathrm{HCV}$-infected patients are in need of a second liver graft. Retransplantation is a controversial issue because of the already limited donor supply and poor outcomes. Retransplantation for recurrent HCV often results in a similar severity of recurrent disease after primary transplantation, and patients often have a higher risk of immediate perioperative mortality [48]. Schluger et al. [4] reported that five of eight patients retransplanted for HCV FCH died of sepsis soon after surgery. Because of the poor outcomes, most transplant programs preclude these patients from repeat transplantation [49].

Kwo et al. $[\bullet 50]$ reported two patients with FCH after liver transplantation who were retransplanted and did well. Both patients were treated with combination IFN 
and ribavirin after recurrent HCV developed in the primary transplant allograft with clearance of HCV RNA from the serum. Nonetheless, both developed graft failure and underwent retransplantation, with the addition of intravenous IFN- $\alpha-2 b$ (5 million units) and subsequent IFN therapy postoperatively. Both patients did well with 36 and 24 months of follow-up, respectively, without recurrence of viremia. Perhaps IFN delivery during the anhepatic phase of transplantation may be a means of preventing recurrent disease after liver transplantation; however, further study is needed.

\section{Conclusions}

FCH after transplantation remains an important clinical problem faced by liver transplant surgeons and hepatologists. Although $\mathrm{FCH}$ occurs in the minority of patients transplanted for HCV, as the number of liver transplants for HCV increases over the next decade, this entity will likely become a more commonly encountered problem. Early recognition and correct diagnosis are critical for patient management because of the progressive onset of liver dysfunction and high incidence of mortality. Other etiologies of cholestasis after transplantation must be ruled out, especially ACR, because the treatment for this condition is to increase immunosuppression, rather than minimize or discontinue it as in FCH. High levels of circulating virus are likely directly cytopathic and immunosuppression appears to play a key role in the pathogenesis of $\mathrm{FCH}$. Other unidentified pathogenic mechanisms may also be important because patients coinfected with HIV/HCV may not have markedly elevated HCV RNA levels, suggesting direct viral injury may not be solely responsible for the damage. Minimizing or even discontinuing immunosuppression can result in a decrease in viral level and possibly ongoing liver injury, but the risk of developing ACR of the liver allograft increases. Additionally, currently no data exist that reduction of immunosuppression improves patient or graft survival.

Combination treatment with IFN and ribavirin may prevent disease progression, but studies evaluating timing, length, and efficacy of therapy are lacking. Other modes of treatment, including DFPP with IFN/ribavirin therapy and intravenous IFN during the anhepatic phase of liver transplantation, were reported to cause reversal of FCH in a few cases. Retransplantation is associated with high morbidity and mortality and is often considered a contraindication in patients with recurrent cholestatic HCV. A better pathologic understanding of this aggressive entity is needed to properly manage this subgroup of patients with progressive cholestatic HCV in immunocompromised states.

\section{Disclosure}

Dr. Gordon has received research grants from and served on the speakers' bureaus for Roche Pharmaceuticals and Schering Plough.

\section{References and Recommended Reading}

Papers of particular interest, published recently, have been highlighted as:

\section{- Of importance}

- Of major importance

1. Feray C, Caccamo L, Alexander GH, et al.: European collaborative study on factors influencing outcome after liver transplanation for hepatitis C. European Concerted Action on Viral Hepatitis (EUROHEP) Group. Gastroenterology 1999, 117:619-625.

2. Berenguer M: Natural history of recurrent hepatitis C. Liver Transpl 2002, 8:S14-S8.

3. Neumann UP, Berg T, Bahra M, et al.: Long-term outcome of liver transplants for chronic hepatitis C: a 10-year follow-up. Transplantation 2004, 77:226-231.

4. Schluger LK, Sheiner PA, Thung SN, et al.: Severe recurrent cholestatic hepatitis $\mathrm{C}$ following orthotopic liver transplantation. Hepatology 1996, 23:971-976.

5. Belli L, Dusheiko G, Rolles K, et al.: Liver transplantation for chronic viral hepatitis. Italian J Gastroent 1991, 23:36.

6. Asanza CG, Garcia-Monzon C, Clemente G, et al.: Immunohistochemical evidence of immunopathogenetic mechanisms in chronic hepatitis $\mathrm{C}$ recurrence after liver transplantation. Hepatology 1997, 26:755.

7. Rosen HR, Gretch DR, Oehlke M, et al.: Timing and severity of initial hepatitis $\mathrm{C}$ recurrence as predictors of long-term liver allograft injury. Transplantation 1998, 65:1178.

8. Ben-Ari Z, Pappo O, Mor E: Intrahepatic cholestasis after liver transplantation. Liver Transpl 2003, 9:1005-1018.

9. Dickson RC, Caldwell SH, Ishitani MB, et al.: Clinical and histologic patterns of early graft failure due to recurrent hepatitis $\mathrm{C}$ in four patients after liver transplantation. Transplantation 1996, 61:701-705.

10. Gane EJ, Portmann BC, Naoumov NV, et al.: Long-term outcome of hepatitis $\mathrm{C}$ infection after liver transplantation. N Engl J Med 1996, 334:815-820.

11. Delgado J, Munoz de Bustillo E, Ibarrola C, et al.: Hepatitis $\mathrm{C}$ virus-related fibrosing cholestatic hepatitis after cardiac transplantation: is azathioprine a contributory factor? J Heart Lung Transplant 1999, 18:607-610.

12. Zylberberg H, Carnot F, Mamzer MF, et al.: Hepatitis $C$ virus-related fibrosing cholestatic hepatitis after renal transplantation. Transplantation 1997, 63:158-160.

13. Delladestima JK, Boletis JN, Makris F, et al.: Fibrosing cholestatic hepatitis in renal transplant recipients with hepatitis c infection. Liver Transpl Surg 1999, 5:294-300.

14. O'Grady JG, Smith HM, Davies SE, et al.: Hepatitis B virus reinfection after orthotopic liver transplantation. Serological and clinical implications. J Hepatol 1992, 14:104-111.

15. Berenguer M, Ferrell L, Watson J, et al.: HCV-related fibrosis progression after liver transplantation: increase in recent years. J Hepatol 2000, 32:673-684.

16. Charlton M, Seaberg E, Wiesner R, et al.: Predictors of patient and graft survival following liver transplantation for hepatitis C. Hepatology 1998, 28:823-830.

17. Sreekumar R, Gonazalez-Koch A, Maor-Kendler Y, et al.: Early identification of recipients with progressive histologic recurrence of hepatitis $\mathrm{C}$ after liver transplantation. Hepatology 2000, 32:1125-1130.

18. Gordon FD, Poterucha JJ, Germer J, et al.: The relationship between hepatitis $\mathrm{C}$ genotype and the severity of recurrent hepatitis $\mathrm{C}$ after liver transplantation. Transplantation 1997, 63:1419-1422.

19. Bernard PH, Le Bail B, Rullier A, et al.: Recurrence and accelerated progression of hepatitis $\mathrm{C}$ after liver transplantation. Semin Liver Dis 2000, 20:533-538.

20. Deshpande V, Burd E, Aardema KL, et al.: High levels of hepatitis $C$ virus RNA in native livers correlate with the development of cholestatic hepatitis in liver allografts and a poor outcome. Liver Transpl 2001, 7:118-124. 
21. Taga SA, Washington MK, Terrault N, et al.: Cholestatic hepatitis C in liver allografts. Liver Transpl Surg 1998, 4:304-310.

22. Pessoa MG, Bzowej N, Berenguer M, et al.: Evolution of hepatitis $C$ virus quasispecies in patients with severe cholestatic hepatitis afterliver transplantation. Hepatology 1999, 30:1513-1520.

23. Doughty AL, Painter DM, McCaughan GW: Post-transplant quasispecies pattern remains stable over time in patients with recurrent cholestatic hepatitis because of hepatitis C virus. J Hepatol 2000, 32:126-134.

24. Xiao SY, Lu L, Wang HL: Fibrosing cholestatic hepatitis: clinicopathologic spectrum, diagnosis, and pathogenesis. Int J Clin Exp Pathol 2008, 1:396-402.

This review article describes the spectrum of clinical conditions in which FCH occurs as well as the histopathologic findings and current understanding of $\mathrm{FCH}$.

25. Davies S, Portmann B, O'Grady J, et al.: Hepatic histological findings after transplantation for chronic hepatitis $B$ infection, including a unique pattern of fibrosing cholestatic hepatitis. Hepatology 1991, 13:150-157.

26.• Dixon LR, Crawford JM: Early histologic changes in fibrosing cholestatic hepatitis C. Liver Transpl 2007, 13:219-226.

This pivotal article highlighted early histologic changes found in

$\mathrm{FCH}$ that had not been identified previously.

27. de Vera ME, Dvorchik I, Tom K, et al.: Survival of liver transplant patients coinfected with HIV and HCV is adversely impacted by recurrent hepatitis C. Am J Transplant 2006, 6:2983-2993.

This important article evaluated the impact of recurrent HCV on 27 HIV-positive patients who underwent liver transplantation compared with a cohort of HIV-negative HCV-positive patients.

28. Staples CT Jr, Rimland D, Dudas D: Hepatitis C in the HIV (human immunodeficiency virus) Atlanta VA (Veterans Affairs Medical Center) Cohort Study (HAVACS): the effect of coinfection on survival. Clin Infect Dis 1999, 29:150-154.

29. Benfield T: Hepatitis C in the EuroSIDA cohort of European HIV-infected patients: prevalence and prognostic value [abstract 22261]. Proceedings of the 12th World AIDS Conference. Geneva, Switzerland; June 28-July 3, 1998.

30. Denis F, Adjide CC, Rogez S, et al.: Seroprevalence of HBV, $\mathrm{HCV}$, and HDV hepatitis markers in 500 patients infected with the human immunodeficiency virus. Pathol Biol (Paris) 1997, 45:701-708.

31. Bica I, McGovern B, Dhar R, et al.: Increasing mortality due to end-stage liver disease in patients with human immunodeficiency virus infection. Clin Infect Dis 2001, 32:492-497.

32. Rosenberg PM, Farrell JJ, Abraczinskas DR, et al.: Rapidly progressive fibrosing cholestatic hepatitis-hepatitis $\mathrm{C}$ virus in HIV coinfection. Am J Gastroenterol 2002, 97:478-483.

33. Neff GW, Shire N, Ruiz P, et al.: The importance of clinical parameters when differentiating cholestatic hepatitis $\mathrm{C}$ virus from allograft rejection. Transplant Proc 2005, 37:4397-4402.

34. Vierling JM, Villamil FG, Rojter SE, et al.: Morbidity and mortality of recurrent hepatitis $\mathrm{C}$ infection after orthotopic liver transplantation. J Viral Hepat 1997, 4(Suppl 1):117-124.

35. Sheiner PA, Schwartz ME, Mor E, et al.: Severe or multiple rejection episodes are associated with early recurrence of hepatitis $\mathrm{C}$ after orthotopic liver transplantation. Hepatology 1995, 21:30-34.

36. Wright HI, Gavaler JS, Van Thiel DH: Preliminary experience with alpha-2b-interferon therapy of viral hepatitis in liver allograft recipients. Transplantation 1992, 53:121-124.
37. Wright TI, Combs C, Kim M, et al.: Interferon alpha therapy for hepatitis $\mathrm{C}$ virus infection after liver transplantation. Hepatology 1994, 20:773-779.

38. Feray C, Habsanne A, Samuel D, et al.: Poor prognosis of patients retransplanted for recurrent liver disease due to hepatitis C virus. J Hepatol 1995, 22:135A.

39. Cattral MS, Krajden M, Wanless IR, et al.: A pilot study of ribavirin therapy for hepatitis $\mathrm{C}$ virus infection after liver transplantation. Transplantation 1996, 61:1483-1488.

40. Gane EJ, Tibbs CJ, Ramage JK, et al.: Ribavirin therapy for hepatitis $\mathrm{C}$ infection following liver transplantation. Transpl Int 1995, 8:61-64.

41. Bizollon T, Palazzo U, Ducerf C, et al.: Pilot study of the combination of interferon alpha and ribavirin as therapy of recurrent hepatitis $\mathrm{C}$ after transplantation. Hepatology 1997, 26:500-504.

42. Gopal DV, Rabkin JM, Berk BS, et al.: Treatment of progressive hepatitis $\mathrm{C}$ recurrence after liver transplantation with combination interferon plus ribavirin. Liver Transpl 2001, 7:181-190.

43. Gopal DV, Rosen HR: Duration of antiviral therapy for cholestatic HCV recurrence may need to be indefinite. Liver Transpl 2003, 9:348-353.

44. Bolkhir A, Brunt EM, Solomon HS, et al.: Sustained resolution of fibrosing cholestatic hepatitis $\mathrm{C}$ despite viremic relapse after stopping pegylated interferon and ribavirin therapy [letter]. Liver Transpl 2007, 13:309-311.

This letter to the editor described a case report raising awareness that discontinuation of combination PEG IFN and ribavirin did not lead to previously reported devastating outcomes in patients with $\mathrm{FCH}$.

45. Chalasani N, Manzarbeitia C, Ferenci P, et al.: Peginterferon alfa-2a for hepatitis $\mathrm{C}$ after liver transplantation: two randomized, controlled trials. Hepatology 2005, 41:289-298.

46. Gordon FD, Kwo P, Vargas HE: Treatment of hepatitis C in liver transplant recipients. Liver Transplantation 2009, 15:126-1235.

This important article reviews the efficacy of combined IFN and ribavirin after recurrent HCV after liver transplantation.

47. Taniguchi M, Furukawa H, Shimamura T, et al.: Impact of double-filtration plasmapheresis in combination with interferon and ribavirin in living donor liver transplant recipients with hepatitic C. Transplantation 2006, 81:1747-1749.

This paper described the successful outcome of using combination IFN and ribavirin with DFPP treatment in patients with $\mathrm{FCH}$.

48. Berenguer M, Prieto M, Palau A, et al.: Severe recurrent hepatitis $\mathrm{C}$ after liver retransplantation for hepatitis C virus-related graft cirrhosis. Liver Transpl 2003, 9:228-235.

49. Burton JR Jr, Androsen HR: Liver retransplantation for hepatitis $\mathrm{C}$ virus recurrence: a survey of liver transplant programs in the United States. Clin Gastroenterol Hepatol 2005, 3:700-704.

50. Kwo PY, Saxena R, Cummings OW: Intravenous interferon during the anhepatic phase of liver retransplantation and prevention of recurrence of cholestatic hepatitis $\mathrm{C}$ virus. Liver Transpl 2007, 13:1710-1713.

This original article described the use of intravenous IFN during the anhepatic phase of liver transplantation with successful prevention of recurrent cholestatic HCV. 